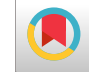

\title{
Possible Nutrition-Related Mechanisms of Metabolic Management in Cancer Treatment
}

\author{
Adeleh Khodabakhshi (iD ${ }^{1,2}$, Maryam Mahmoudi ${ }^{3}$, Hassan Mehrad Majd ${ }^{4}$ and Sayed Hossein Davoodi \\ (iD ${ }^{2, *}$ \\ ${ }^{1}$ Department of Nutrition, Faculty of Public Health, Kerman University of Medical Sciences, Kerman, Iran \\ ${ }^{2}$ Cancer Research Center, Shahid Beheshti University of Medical Sciences, Tehran, Iran \\ ${ }^{3}$ Department of Cellular and Molecular Nutrition, School of Nutritional Sciences and Dietetics, Tehran University of Medical Sciences, Tehran, Iran \\ ${ }^{4}$ Cancer Molecular Pathology Research Center, Mashhad University of Medical Sciences, Mashhad, Iran \\ "Corresponding author: Cancer Research Center, Shahid Beheshti University of Medical Sciences, Tehran, Iran. Email: hdavoodi1345@gmail.com
}

Received 2020 July 19; Revised 2020 October 26; Accepted 2020 October 26

\begin{abstract}
Context: Somatic mutation theory has been considered as a potential cause for cancer. However, major inconsistencies with the gene theory have necessitated serious reconsideration of this assumption. According to these inconsistencies, cancer may be considered as a metabolic disorder. According to the mitochondrial metabolic theory, substrate-level phosphorylation has been suggested to be superior to oxidative phosphorylation in cancer cells. Cancer metabolic therapies such as ketogenic diets (KD) and limitation in glutamine and calorie can be beneficial and are in line with this theory. In this study, we have reviewed the potential effects of KD as well as glutamine and calorie restriction in various types/stages of cancer with a focus on possible mechanisms.

Evidence Acquisition: A comprehensive electronic search of different databases was performed using "cancer", "ketogenic diet", and "metabolic" as the main keywords. A comprehensive electronic search of different databases was performed using "cancer", "ketogenic diet", and "metabolic" as the main keywords.

Results: Emerging evidence has indicated that KD can affect tumor cells by reducing glucose availability and simultaneous elevation of ketone bodies as non-fermentable metabolic fuels. KD has been suggested to be more effective as a non-toxic therapeutic measure in combination with glutamine targeting agents, chloroquine for lysosomal targeting, hyperbaric oxygen therapy, and calorie restriction.
\end{abstract}

Conclusions: This metabolic approach can be considered as a promising non-toxic strategy for cancer management.

Keywords: Cancer, Ketogenic Diet, Glycolysis, Glucose, Glutamine, Non-Toxic, Metabolic, Hyperbaric Oxygenation

\section{Context}

Many of the current cancer treatments have considered cancer as a genetic disorder. However, major inconsistencies with the gene theory have necessitated serious reconsideration of this assumption. For instance, the absence of gene and chromosomal mutations in some cancers that reinforce the somatic mutation theory (1$4)$; driver gene mutations in various normal human tissues including the breast (5-7); and lack of various cancers in chimpanzees despite the large similarity in gene sequence with humans are some pieces of evidence indicating this inconsistency (8-10). Moreover, nuclear/cytoplasm transfer experiments have shown the possibility of normal cell and tissue production from tumorigenic nuclei relocated in normal mitochondria containing cytoplasm (11). Recent reports have shown that multiple oncogenic pathways and growth behavior can be down-regulated in metastatic breast cancer cells by normal mitochondria (12, 13). These findings indicate that normal mitochondrial function plays a vital role in suppressing tumorigenesis regardless of the tumor nucleus gene or chromosomal abnormalities. These findings also suggest that the nuclear genome mutations are not the primary cause of cancer. Cancer was suggested as a metabolic disease that is caused by an irreversible damage to cellular respiration. Cancer cells heavily depend on glucose fermentation to lactate for their metabolic demands even under sufficient oxygen supply (14). The glucose transporter molecules on tumor cell surfaces have shown a substantial up-regulation. Also, some glycolytic enzymes including hexokinase 2, lactate dehydrogenase-A, phosphofructokinase, and pyruvate kinase-M2 have been reported to be overexpressed. Abnormal function of the tricarboxylic acid (TCA) cycle enzymes 
in cancer cells cause a remarkable reduction in ATP production via decreased oxidative phosphorylation and enhanced glycolysis $(15,16)$. The secretion of lactic acid increases during increased glycolysis and can decrease the extracellular $\mathrm{pH}$ around the tumor. This acidosis induces normal cell death, enhances angiogenesis, deteriorates extracellular matrix, and suppresses tumor antigen-specific immune responses which promote metastasis. Consequently, the tumors become more aggressive and desmoplastic (17). Also, glycolysis generates NADPH via its pentose phosphate pathway offshoot, which produces glutathione that decreases reactive oxygen species-induced death in cancer cells (18). Also, carbon chains in cancer cells are used as precursors to produce essential cell proliferation materials including nucleic acids, proteins, and lipids (19). In light of these findings, cancer is a chronic systemic disease with a strong metabolic peculiarity that theoretically provides an ideal target for metabolic therapies.

Several examples of metabolic therapeutic strategies for cancer treatment are presented in this review study. This article has focused on the ketogenic diet (KD) and other metabolic therapies that increase the effectiveness of $\mathrm{KD}$ in metabolic treatment of cancer.

\section{Evidence Acquisition}

A comprehensive review of electronic databases including ISI web of knowledge, Scopus, PubMed, and Google Scholar using the main keywords of "cancer", "ketogenic diet", and "metabolic" was performed considering the published manuscripts until the end of June 2020. A manual search among the references of gathered articles was also performed to improve the precision of the review.

\section{Results}

\subsection{Ketogenic Diet}

Ketogenic diet high in fats and low in glucose, is an effective complementary and alternative therapeutic approach for managing a variety of human cancers. Since the number, structure, and function of the mitochondria and subsequently, oxidative phosphorylation in cancer cells are defective, the use of fatty acids and ketone bodies for ATP synthesis cannot be performed $(20,21)$. These diets lead to cell starvation and energy deprivation in cancer cells while normal cells can use either glucose or ketone bodies and survive. Besides, reduced levels of blood glucose decreases insulin and insulin-like growth factor (IGF) which are important players in cancer cell growth and proliferation (22). Decreased levels of glucose will also favors the reduction in substrates required in both glycolytic and the pentose phosphate ( $\mathrm{PP}$ ) pathways which in turn leads to a decrease in cellular energy, and the synthesis of glutathione and nucleotide precursors.

Also it was demonstrated that under Ketogenic diets the hypoxic, acidotic, and glucose and glutamine enriched pro-tumorigenic microenvironment of tumor cells might become less inflamed (23-25). Ketogenic diets are associated with reduced cellular proliferation, impeded tumor growth, reduced inflammation, neovascularization and angiogenesis, and increased apoptosis (26). The underlying mechanisms have not yet been fully characterized. During tumor progression, many angiogenic activators such as interleukin 8 (IL-8), tumor necrosis factor a (TNF-a), hypoxiainducible factors (HIFs), and vascular endothelial growth factors (VEGFs) support the process of angiogenesis. KD or caloric restriction in mouse glioma models dissuade the formation of tumor microvasculature accompanied by a significant reduced levels of HIF-1a and VEGF receptor. The anti-inflammatory effects of KD are performed by suppressing the activation of NLRP3 inflammasome and reduction of inflammatory factors like TNF-a, interleukin 1 (IL-1), interleukin 6 (IL-6), and interleukin 18 (IL-18), and prostaglandin E2 (PGE2).

Even at the epigenetic level, ketogenic diet and fatty acids inhibit Histone deacetylases (HDAC) enzyme and affect methylation $(27,28)$. RASSF1A as a tumor-suppressor gene has been reported to be epigenetically inactivated at high frequency in various cancer tissues. Also DNAmethylation of HIN1 gene promoter frequently occurs in breast cancer. Epigenetic silencing of HIN1 expression induces breast cancer cell growth, migration, and invasion. Any restoration in HIN1 expression due to the use of ketogenic diet can help to suppress cancer cells growth. The Ki67 is a cell proliferation activity marker that correlates with the clinical course and prognosis of tumors. Obviously, the use of DNA methyltransferase inhibitors may represent a potential therapeutic strategy for breast cancer treatment. In totally, KD seems to promote its anti-proliferative effect on cancer cells by creating an unfavorable metabolic environment.

\subsection{Clinical Studies}

While, a large number of animal studies have provided evidence for anti-tumor effects of KDs (29), support for these effects is very limited in humans. Studies involving children or adults affected with cancer have demonstrated the safety and tolerability of ketogenic diets. These studies are also motivating clinical trial. To the best of our knowledge only 3 randomized controlled trials have been conducted on the anti-cancer effects of the ketogenic diet.

In our previous study we reported that chemotherapy combined with 12-week KDs might exert beneficial effects 
by decreasing total body fat, TNF- $\alpha$, and insulin as well as increasing IL-10. KD may lead to reductions in tumor size and down-staging in patients with breast cancer. KD can also improve the overall survival without any substantial side effects on the biochemical parameters and quality of life (30-32).

In another trial a significant between-group difference was reported in adjusted physical function scores, cravings for starchy foods and fast food, body fat and insulin between the patients with ovarian or endometrial cancer undergoing 12 weeks of $\mathrm{KD}$ and the control group $(33,34)$.

Also, compared to a standard diet (SD), low carbohydrate or ketogenic diets have been reported to improve the quality of life, physical performance, body composition, and metabolic health in patients with breast cancer (35).

These feasible and tolerable dietary approaches might improve the oncological outcomes and has the potential to improve the therapeutic response to medications that have been well documented in vitro and in vivo. However, further randomized clinical trials are needed to confirm these data.

\subsection{Restricted Calorie}

Restricted ketogenic diets can reduce glucose and elevate blood ketone bodies more effectively than calorie restriction or KD alone. Calorie restricted ketogenic diets induce both chronic and intermittent acute stress on the energy metabolism of tumor cell, while simultaneously protect and enhance the normal cell energy metabolism.

The favorable effects of fasting in chronic disease have been reported in several previous studies as reduced calorie intake provides protection against oxidative stress and aging in various organisms.

Fasting has been proposed to promote substantial changes in metabolic pathways and cellular processes including autophagy and stress response, as well as decreasing the IGF-1. As a result, other factors as Akt, Ras, and mTOR will be affected and will down-regulate cell growth and proliferation (36).

The anti-angiogenic, anti-inflammatory, and proapoptotic effects of fasting and dietary restriction target multiple cancer hallmarks (37-40) which enhances the efficacy of chemo- and radiation therapy and reduces the side effects. Hence, lower dosages of chemotherapeutic drugs can be used in adjuvant therapy with calorie restriction or ketogenic diets.

The existing data support the safety and feasibility of these approaches and suggest an improvement in the quality of life and fatigue of patients under chemotherapy (41, 42). In another randomized trial in 13 patients with breast cancer, demonstrated no significant differences in the toxicity resulted from chemotherapy in patients undergoing fasting, while DNA damages on peripheral blood mononuclear cells were significantly reduced during the first 30 minutes after intervention in fasting patients (43). However, data regarding the effects of this approach on patient survival outcomes are still controversial and need to be considered more in future studies.

\subsection{Targeting Glutamine for Metastatic Cancer}

While glucose serve as a prime fuel for growth and development of various tumors, some tumors use glutamine (44-47). Glutamine targeting in glutamine-dependent cancer cells can be a novel potential therapeutic approach. Glutamine plays several important roles in various metabolic pathways. Its amide nitrogen is used for nucleotide synthesis. Also, the glutamine-derived glutamate is used in protein synthesis by providing anapleurotic carbons to the Krebs cycle through alpha-ketoglutarate (alpha-KG). It also participates in ATP synthesis through the TCA cycle (48). Tumor cells in hypoxic conditions can use the glutamine-derived ATP from the Krebs cycle. Excess glutamine can also promote cell growth and suppress autophagy through stimulating the activity of a cell signaling pathway called serine/threonine kinase mammalian target of rapamycin complex 1 (mTORC1) $(49,50)$.

Glutaminolysis is performed by glutaminase (GLS) which is upregulated in several cancers. The glutaminase inhibitor DON (6-diazo-5-oxo-L-norleucine) has shown clinical benefits $(45,51)$. It could be more effective in combination with glycolysis inhibitors and/or calorie restriction (52). DON has been shown to be effective in reducing both primary tumor size and systemic metastasis (53).

Since glutamine is involved in various cellular metabolic functions especially in the immune system, glutamine targeting must be more emphasized regarding its possible side effects than glucose targeting. As metastatic cells with characteristics of macrophages and other immune cells are more dependent on glutamine as a major fuel, then glutamine targeting should be an effective therapeutic strategy in reducing most metastatic cancers (54).

In addition, accumulating evidence suggests that glutamine metabolism is regulated by many factors including the origin, suppressor status, epigenetic alternations, and microenvironment of the tumor (55). These concerns should better be considered in developing dietary based glutamine targeting tumor therapy. The specific mechanisms mediating tumor cell adaptation to glutamine limitation also need to be defined (56).

\subsection{Lysosomal Digestion}

Phagocytosed glycoconjugates and proteins can undergo lysosomal digestion and generate glucose and glu- 
tamine. It has been shown that glioblastoma cells with myeloid properties are able to survive in extracellular matrix material without any additive glucose and glutamine (57).

Cumulative concentration of lactate in the media was indicative of lysosomal digestion and aerobic fermentation of glycoconjugates in the extracellular matrix material. However, the glioblastoma cells died immediately after adding chloroquine that stops the lysosomal digestion (57) A similar mechanism has been reported in pancreatic ductal adenocarcinoma cells under low nutrient conditions (58). Hence, targeting the lysosomal digestion in low glucose and glutamine conditions can inhibit metastatic invasive tumors.

\subsection{Ketogenic Diet in Combination with Hyperbaric Oxygen Therapy}

Hyperbaric oxygen therapy (HBOT) has been demonstrated to enhance the ability of $\mathrm{KD}$ in reducing tumor proliferation and metastasis. Although the evidence from both animal and human studies support the anti-cancer effect of hyperbaric oxygen therapy (59). The effectiveness will be enhanced when it is combined with standard care prior to radiation therapy for glioblastoma multiform (60). However, the exact mechanism through which of hyperbaric oxygen affects the tumor is not clear yet. Hyperbaric oxygen has been suggested to reverse hypoxia and suppress tumor growth (61) and Hyperbaric oxygen therapy enhances oxidative stress and peroxidation of lipids in glioblastoma multiform cell membrane (62). Also, exogenous ketones can enhance the effects of the ketogenic diet and hyperbaric oxygen therapy (63).

Although hyperbaric oxygen and radiotherapy both induce oxidative stress and kill tumor cells, normal cells are more compatible with the former one.

Consistent with findings of previous case report studies $(64,65)$, a complete response has been reported with a 6-month combination of chemotherapy, ketogenic diet, hyperthermia, and hyperbaric oxygen therapy in a patient with breast cancer (66).

Thus, the combined use of KD along with other metabolic approaches and standard therapy in order to enhance the therapeutic response in cancer can be used to design clinical trials for non-toxic management of most cancers.

\section{Conclusions}

Glucose and glutamine restriction along with increasing the non-fermentable ketones can be a potential complementary strategy for cancer treatment. Also, targeting the lysosomal digestion through the administration of chloroquine and hyperbaric oxygen combined with a ketogenic diet will kill tumor cells. Data from further prospective randomized trials are needed to confirm these data.

\section{Acknowledgments}

We would like to thank Clinical Research Development Center of Ghaem Hospital, Mashhad University of Medical Sciences, for their assistance in this manuscript.

\section{Footnotes}

Authors' Contribution: Adeleh Khodabakhshi and Hossein Davoodi did search. Adeleh Khodabakhshi wrote manuscript. Maryam Mahmoudi rewrote manuscript. Hassan Mehrad Majd did critical review.

Conflict of Interests: The authors declare that they have no conflict of interests.

Funding/Support: Not applicable.

\section{References}

1. Baker SG. A cancer theory kerfuffle can lead to new lines of research. J Natl Cancer Inst. 2015;107(2). doi: 10.1093/jnci/dju405. [PubMed: 25528755]. [PubMed Central: PMC4326310].

2. Soto AM, Sonnenschein C. The somatic mutation theory of cancer: growing problems with the paradigm? Bioessays. 2004;26(10):1097107. doi: 10.1002/bies.20087. [PubMed: 15382143].

3. Bayreuther K. Chromosomes in primary neoplastic growth. Nature. 1960;186:6-9. doi: 10.1038/186006a0. [PubMed: 13797839].

4. Seyfried TN, Mukherjee P, Iyikesici MS, Slocum A, Kalamian M, Spinosa JP, et al. Consideration of Ketogenic Metabolic Therapy as a Complementary or Alternative Approach for Managing Breast Cancer. Front Nutr. 2020;7:21. doi: 10.3389/fnut.2020.00021. [PubMed: 32219096]. [PubMed Central: PMC7078107].

5. Martincorena I, Campbell PJ. Somatic mutation in cancer and normal cells. Science.2015;349(6255):1483-9. doi:10.1126/science.aab4082. [PubMed: 26404825].

6. Chanock SJ. The paradox of mutations and cancer. Science. 2018;362(6417):893-4. doi: 10.1126/science.aav5697. [PubMed: 30467157].

7. Martincorena I, Fowler JC, Wabik A, Lawson ARJ, Abascal F, Hall MWJ, et al. Somatic mutant clones colonize the human esophagus with age. Science. 2018;362(6417):911-7. doi: 10.1126/science.aau3879. [PubMed: 30337457]. [PubMed Central: PMC6298579].

8. Huttley GA, Easteal S, Southey MC, Tesoriero A, Giles GG, McCredie MR, et al. Adaptive evolution of the tumour suppressor BRCA1 in humans and chimpanzees. Australian Breast Cancer Family Study. Nat Genet. 2000;25(4):410-3. doi:10.1038/78092. [PubMed:10932184].

9. van den Berg RA, Hoefsloot HC, Westerhuis JA, Smilde AK, van der Werf MJ. Centering, scaling, and transformations: improving the biological information content of metabolomics data. BMC Genomics. 2006;7:142. doi: 10.1186/1471-2164-7-142. [PubMed: 16762068]. [PubMed Central: PMC1534033].

10. Varki NM, Varki A. On the apparent rarity of epithelial cancers in captive chimpanzees. Philos Trans R Soc Lond B Biol Sci. 2015;370(1673). doi: 10.1098/rstb.2014.0225. [PubMed: 26056369]. [PubMed Central: PMC4581030]. 
11. Seyfried TN. Cancer as a mitochondrial metabolic disease. Front Cell Dev Biol. 2015;3:43. doi: 10.3389/fcell.2015.00043. [PubMed: 26217661]. [PubMed Central: PMC4493566].

12. Kaipparettu BA, Ma Y, Park JH, Lee TL, Zhang Y, Yotnda P, et al. Crosstalk from non-cancerous mitochondria can inhibit tumor properties of metastatic cells by suppressing oncogenic pathways. PLoS One. 2013;8(5). e61747. doi: 10.1371/journal.pone.0061747. [PubMed: 23671572]. [PubMed Central: PMC3650012].

13. Chang JC, Chang HS, Wu YC, Cheng WL, Lin TT, Chang HJ, et al. Mitochondrial transplantation regulates antitumour activity, chemoresistance and mitochondrial dynamics in breast cancer. J Exp Clin Cancer Res. 2019;38(1):30. doi: 10.1186/s13046-019-1028-z. [PubMed: 30674338]. [PubMed Central: PMC6343292].

14. Warburg O. On the origin of cancer cells. Science. 1956;123(3191):30914. doi:10.1126/science.123.3191.309. [PubMed: 13298683].

15. Moreno-Sanchez R, Rodriguez-Enriquez S, Marin-Hernandez A, Saavedra E. Energy metabolism in tumor cells. FEBS J. 2007;274(6):1393-418. doi: 10.1111/j.1742-4658.2007.05686.x. [PubMed:17302740].

16. Deberardinis RJ, Sayed N, Ditsworth D, Thompson CB. Brick by brick: metabolism and tumor cell growth. Curr Opin Genet Dev. 2008;18(1):54-61. doi: 10.1016/j.gde.2008.02.003. [PubMed: 18387799]. [PubMed Central: PMC2476215].

17. Gatenby RA, Gawlinski ET, Gmitro AF, Kaylor B, Gillies RJ. Acidmediated tumor invasion: a multidisciplinary study. Cancer Res. 2006;66(10):5216-23. doi: 10.1158/0008-5472.CAN-05-4193. [PubMed: 16707446].

18. King A, Gottlieb E. Glucose metabolism and programmed cell death: an evolutionary and mechanistic perspective. Curr Opin Cell Biol. 2009;21(6):885-93. doi: 10.1016/j.ceb.2009.09.009. [PubMed: 19850457 .

19. Kroemer G, Pouyssegur J. Tumor cell metabolism: cancer's Achilles' heel. Cancer Cell. 2008;13(6):472-82. doi: 10.1016/j.ccr.2008.05.005. [PubMed: 18538731].

20. Seyfried TN, Yu G, Maroon JC, D'Agostino DP. Press-pulse: a novel therapeutic strategy for the metabolic management of cancer. Nutr Metab (Lond). 2017;14:19. doi: 10.1186/s12986-017-0178-2. [PubMed: 28250801]. [PubMed Central: PMC5324220].

21. Chinopoulos C, Seyfried TN. Mitochondrial Substrate-Level Phosphorylation as Energy Source for Glioblastoma: Review and Hypothesis. ASN Neuro. 2018;10:1759091418818260. doi: 10.1177/1759091418818261. [PubMed: 30909720]. [PubMed Central: PMC6311572].

22. Weber DD, Aminazdeh-Gohari S, Kofler B. Ketogenic diet in cancer therapy. Aging (Albany NY). 2018;10(2):164-5. doi: 10.18632/aging.101382. [PubMed: 29443693]. [PubMed Central: PMC5842847].

23. Seyfried TN, Shelton L, Arismendi-Morillo G, Kalamian M, Elsakka A, Maroon J, et al. Provocative Question: Should Ketogenic Metabolic Therapy Become the Standard of Care for Glioblastoma? Neurochem Res. 2019;44(10):2392-404. doi: 10.1007/s11064-019-02795-4. [PubMed: 31025151].

24. Mulrooney TJ, Marsh J, Urits I, Seyfried TN, Mukherjee P. Influence of caloric restriction on constitutive expression of NF-kappaB in an experimental mouse astrocytoma. PLoS One. 2011;6(3). e18085. doi: 10.1371/journal.pone.0018085. [PubMed: 21479220]. [PubMed Central: PMC3068150].

25. Urits I, Mukherjee P, Meidenbauer J, Seyfried TN. Dietary restriction promotes vessel maturation in a mouse astrocytoma. J Oncol. 2012;2012:264039. doi: 10.1155/2012/264039. [PubMed: 22253625]. [PubMed Central: PMC3255299].

26. Wright C, Simone NL. Obesity and tumor growth: inflammation, immunity, and the role of a ketogenic diet. Curr Opin Clin Nutr Metab Care. 2016;19(4):294-9. doi: 10.1097/MCO.0000000000000286. [PubMed: 27168354].

27. Kobow K, Kaspi A, Harikrishnan KN, Kiese K, Ziemann M, Khurana I, et al. Deep sequencing reveals increased DNA methylation in chronic rat epilepsy. Acta Neuropathol. 2013;126(5):741-56. doi:10.1007/s00401013-1168-8. [PubMed: 24005891]. [PubMed Central: PMC3825532].
28. Davie JR. Inhibition of histone deacetylase activity by butyrate. J Nutr. 2003;133(7 Suppl):2485S-93S. doi: 10.1093/jn/133.7.2485S. [PubMed: 12840228].

29. Klement RJ. Beneficial effects of ketogenic diets for cancer patients: a realist review with focus on evidence and confirmation. Med Oncol. 2017;34(8):132. doi: 10.1007/s12032-017-0991-5. [PubMed: 28653283].

30. Khodabakhshi A, Akbari ME, Mirzaei HR, Seyfried TN, Kalamian M, Davoodi SH. Effects of Ketogenic metabolic therapy on patients with breast cancer: A randomized controlled clinical trial. Clin Nutr. 2020. doi: 10.1016/j.clnu.2020.06.028. [PubMed: 32703721].

31. Khodabakhshi A, Akbari ME, Mirzaei HR, Mehrad-Majd H, Kalamian M, Davoodi SH. Feasibility, Safety, and Beneficial Effects of MCTBased Ketogenic Diet for Breast Cancer Treatment: A Randomized Controlled Trial Study. Nutr Cancer. 2020;72(4):627-34. doi: 10.1080/01635581.2019.1650942. [PubMed: 31496287].

32. Khodabakhshi A, Seyfried TN, Kalamian M, Beheshti M, Davoodi SH. Does a ketogenic diet have beneficial effects on quality of life, physical activity or biomarkers in patients with breast cancer: a randomized controlled clinical trial. Nutr J. 2020;19(1):87. doi: 10.1186/s12937-02000596-y. [PubMed: 32828130]. [PubMed Central: PMC7443288].

33. Cohen CW, Fontaine KR, Arend RC, Alvarez RD, Leath CI, Huh WK, et al. A Ketogenic Diet Reduces Central Obesity and Serum Insulin in Women with Ovarian or Endometrial Cancer.J Nutr. 2018;148(8):125360. doi: 10.1093/jn/nxy119. [PubMed: 30137481].

34. Cohen CW, Fontaine KR, Arend RC, Soleymani T, Gower BA. Favorable Effects of a Ketogenic Diet on Physical Function, Perceived Energy, and Food Cravings in Women with Ovarian or Endometrial Cancer: A Randomized, Controlled Trial. Nutrients. 2018;10(9). doi: 10.3390/nu10091187. [PubMed: 30200193]. [PubMed Central: PMC6163837].

35. Kämmerer U, Klement RJ, Sütterlin M, Reuss-Borst M. Low Carb (LCD) and Ketogenic (KD) Diets Increase Quality of Life, Physical Performance, Body Composition and Metabolic Health of Women with Breast Cancer Better Than a Standard Diet (SD). Res Square. 2020. doi: 10.21203/rs.3.rs-28430/v1.

36. Safdie FM, Dorff T, Quinn D, Fontana L, Wei M, Lee C, et al. Fasting and cancer treatment in humans: A case series report. Aging (Albany NY). 2009;1(12):988-1007. doi: 10.18632/aging.100114. [PubMed: 20157582]. [PubMed Central: PMC2815756].

37. Marsh J, Mukherjee P, Seyfried TN. Akt-dependent proapoptotic effects of dietary restriction on late-stage management of a phosphatase and tensin homologue/tuberous sclerosis complex 2-deficient mouse astrocytoma. Clin Cancer Res. 2008;14(23):7751-62. doi: 10.1158/1078-0432.CCR-08-0213. [PubMed: 19047102].

38. Mukherjee P, Abate LE, Seyfried TN. Antiangiogenic and proapoptotic effects of dietary restriction on experimental mouse and human brain tumors. Clin Cancer Res. 2004;10(16):5622-9. doi: 10.1158/10780432.CCR-04-0308. [PubMed: 15328205].

39. Zhou W, Mukherjee P, Kiebish MA, Markis WT, Mantis JG, Seyfried TN. The calorically restricted ketogenic diet, an effective alternative therapy for malignant brain cancer. Nutr Metab (Lond). 2007;4:5. doi: 10.1186/1743-7075-4-5. [PubMed: 17313687]. [PubMed Central: PMC1819381].

40. Mukherjee P, Mulrooney TJ, Marsh J, Blair D, Chiles TC, Seyfried TN. Differential effects of energy stress on AMPK phosphorylation and apoptosis in experimental brain tumor and normal brain. Mol Cancer. 2008;7:37. doi: 10.1186/1476-4598-7-37. [PubMed: 18474106]. [PubMed Central: PMC2397440].

41. Bauersfeld SP, Kessler CS, Wischnewsky M, Jaensch A, Steckhan N, Stange R, et al. The effects of short-term fasting on quality of life and tolerance to chemotherapy in patients with breast and ovarian cancer: a randomized cross-over pilot study. BMC Cancer. 2018;18(1):476. doi:10.1186/s12885-018-4353-2. [PubMed: 29699509]. [PubMed Central: PMC5921787].

42. Dorff TB, Groshen S, Garcia A, Shah M, Tsao-Wei D, Pham H, et al. 
Safety and feasibility of fasting in combination with platinum-based chemotherapy. BMC Cancer. 2016;16:360. doi: 10.1186/s12885-016-23706. [PubMed: 27282289]. [PubMed Central: PMC4901417].

43. de Groot S, Vreeswijk MP, Welters MJ, Gravesteijn G, Boei JJ, Jochems A, et al. The effects of short-term fasting on tolerance to (neo) adjuvant chemotherapy in HER2-negative breast cancer patients: a randomized pilot study. BMC Cancer. 2015;15:652. doi: 10.1186/s12885-0151663-5. [PubMed: 26438237]. [PubMed Central: PMC4595051].

44. Medina MA. Glutamine and cancer. J Nutr. 2001;131(9 Suppl):2539S42S. discussion 2550S-1S. doi: 10.1093/jn/131.9.2539S. [PubMed: 11533309].

45. Cervantes-Madrid D, Romero Y, Duenas-Gonzalez A. Reviving Lonidamine and 6-Diazo-5-oxo-L-norleucine to Be Used in Combination for Metabolic Cancer Therapy. Biomed Res Int. 2015;2015:690492. doi: 10.1155/2015/690492. [PubMed: 26425550]. [PubMed Central: PMC4575731].

46. Dang CV. Glutaminolysis: supplying carbon or nitrogen or both for cancer cells? Cell Cycle. 2010;9(19):3884-6. doi: 10.4161/cc.9.19.13302. [PubMed: 20948290].

47. Reitzer LJ, Wice BM, Kennell D. Evidence that glutamine, not sugar, is the major energy source for cultured HeLa cells. J Biol Chem. 1979;254(8):2669-76. [PubMed: 429309].

48. Chinopoulos $\mathrm{C}$. Which way does the citric acid cycle turn during hypoxia? The critical role of alpha-ketoglutarate dehydrogenase complex. J Neurosci Res. 2013;91(8):1030-43. doi: 10.1002/jnr.23196. [PubMed: 23378250].

49. DeBerardinis RJ, Cheng T.Q's next: the diverse functions of glutamine in metabolism, cell biology and cancer. Oncogene. 2010;29(3):31324. doi: 10.1038/onc.2009.358. [PubMed: 19881548]. [PubMed Central: PMC2809806].

50. Nicklin P, Bergman P, Zhang B, Triantafellow E, Wang H, Nyfeler $B$, et al. Bidirectional transport of amino acids regulates mTOR and autophagy. Cell.2009;136(3):521-34. doi:10.1016/j.cell.2008.11.044. [PubMed: 19203585]. [PubMed Central: PMC3733119].

51. Mueller C, Al-Batran S, Jaeger E, Schmidt B, Bausch M, Unger $\mathrm{C}$, et al. A phase IIa study of PEGylated glutaminase (PEG-PGA) plus 6-diazo-5-oxo-L-norleucine (DON) in patients with advanced refractory solid tumors. J Clin Oncol. 2008;26(15_suppl):2533. doi: 10.1200/jco.2008.26.15_suppl.2533.

52. Seyfried TN. Cancer as a metabolic disease: On the origin, management, and prevention of cancer. John Wiley \& Sons; 2012. doi: $10.1002 / 9781118310311$.

53. Shelton LM, Huysentruyt LC, Seyfried TN. Glutamine targeting inhibits systemic metastasis in the VM-M3 murine tumor model. Int J Cancer. 2010;127(10):2478-85. doi: 10.1002/ijc.25431. [PubMed: 20473919]. [PubMed Central: PMC2946425].

54. Huysentruyt LC, Seyfried TN. Perspectives on the mesenchymal origin of metastatic cancer. Cancer Metastasis Rev. 2010;29(4):695-707. doi: 10.1007/s10555-010-9254-z. [PubMed: 20839033]. [PubMed Central: PMC2962789].

55. Jiang J, Srivastava S, Zhang J. Starve Cancer Cells of Glutamine: Break the Spell or Make a Hungry Monster? Cancers (Basel). 2019;11(6). doi: 10.3390/cancers11060804. [PubMed: 31212591]. [PubMed Central: PMC6627209].

56. Wappler J, Arts M, Roth A, Heeren RMA, Peter Neumann U, Olde Damink SW, et al. Glutamine deprivation counteracts hypoxiainduced chemoresistance. Neoplasia. 2020;22(1):22-32. doi 10.1016/j.neo.2019.10.004. [PubMed: 31765939]. [PubMed Central: PMC6883317].

57. Shelton LM. Targeting energy metabolism in brain cancer. Chestnut Hill: Boston College; 2010.

58. Kamphorst JJ, Nofal M, Commisso C, Hackett SR, Lu W, Grabocka E, et al. Human pancreatic cancer tumors are nutrient poor and tumor cells actively scavenge extracellular protein. Cancer Res. 2015;75(3):544-53. doi: 10.1158/0008-5472.CAN-14-2211. [PubMed: 25644265]. [PubMed Central: PMC4316379].

59. Raffaghello L, Lee C, Safdie FM, Wei M, Madia F, Bianchi G, et al. Starvation-dependent differential stress resistance protects normal but not cancer cells against high-dose chemotherapy. Proc Natl Acad Sci U S A. 2008;105(24):8215-20. doi: 10.1073/pnas.0708100105. [PubMed: 18378900]. [PubMed Central: PMC2448817].

60. Kohshi K, Beppu T, Tanaka K, Ogawa K, Inoue O, Kukita I, et al. Potential roles of hyperbaric oxygenation in the treatments of brain tumors. Undersea Hyperb Med. 2013;40(4):351-62. [PubMed: 23957206].

61. Moen I, Stuhr LE. Hyperbaric oxygen therapy and cancer-a review. Target Oncol. 2012;7(4):233-42. doi: 10.1007/s11523-012-0233-x. [PubMed: 23054400]. [PubMed Central: PMC3510426].

62. D'Agostino DP, Colomb DJ, Dean JB. Effects of hyperbaric gases on membrane nanostructure and function in neurons. J Appl Physiol (1985). 2009;106(3):996-1003. doi: 10.1152/japplphysiol.91070.2008. [PubMed: 18818382].

63. Poff AM, Ward N, Seyfried TN, Arnold P, D'Agostino DP. NonToxic Metabolic Management of Metastatic Cancer in VM Mice: Novel Combination of Ketogenic Diet, Ketone Supplementation, and Hyperbaric Oxygen Therapy. PLoS One. 2015;10(6). e0127407. doi: 10.1371/journal.pone.0127407. [PubMed: 26061868]. [PubMed Central: PMC4464523].

64. Iyikesici MS, Slocum AK, Slocum A, Berkarda FB, Kalamian M, Seyfried TN. Efficacy of Metabolically Supported Chemotherapy Combined with Ketogenic Diet, Hyperthermia, and Hyperbaric Oxygen Therapy for Stage IV Triple-Negative Breast Cancer. Cureus. 2017;9(7). e1445. doi: 10.7759/cureus.1445. [PubMed: 28924531]. [PubMed Central: PMC5589510].

65. Elsakka AMA, Bary MA, Abdelzaher E, Elnaggar M, Kalamian M, Mukherjee P, et al. Management of Glioblastoma Multiforme in a Patient Treated With Ketogenic Metabolic Therapy and Modified Standard of Care: A 24-Month Follow-Up. Front Nutr. 2018;5:20 doi: 10.3389/fnut.2018.00020. [PubMed: 29651419]. [PubMed Central: PMC5884883].

66. Iyikesici MS. Feasibility study of metabolically supported chemotherapy with weekly carboplatin/paclitaxel combined with ketogenic diet, hyperthermia and hyperbaric oxygen therapy in metastatic non-small cell lung cancer. Int J Hyperthermia. 2019;36(1):446-55. doi: 10.1080/02656736.2019.1589584. [PubMed: 30931666]. 